\title{
A firamework for e-cooperating business agents: An application to the (re)engineering of production facilities
}

\author{
Gil Manuel Gonçalves, João Borges de Sousa, Fernando Lobo Pereira, Paulo Sousa \\ Dias, António Santos \\ Department of electrical and Computer Engineering, Faculdade de Engenharia da \\ Universidade do Porto, Rua Dr. Roberto Frias, 4200 - 465 Porto, Portugal. \\ (\{gil, jtasso, flp, pdias, ansantos\}@fe.up.pt)
}

\begin{abstract}
The task of coordinating business agents to accomplish complex goals, which could not be accomplished by any of the agents on its own, is a complex one. This paper addresses the problem of coordinating companies within a general architecture for e-cooperation. This architecture articulates the interoperation of business process over the Internet with varied degrees of security requirements. The implementation infrastructure is used to support the cooperative design and construction of production facilities (spot-welding lines, assembly lines). These are typically complex systems, which are made of several subsystems (robots, machines, cells), that are designed and built by different companies in different countries. Design and construction involves several types of companies, from OEMs to small engineering houses. With this architecture we aim at improving the otherwise time consuming task of coordinating their activities, especially when models (geometric, process, and workflow models), machine programs, documentation, etc. must be exchanged frequently and securely.
\end{abstract}
Keywords
Cooperative Work, Cooperative Systems and Technologies, Workflow Systems and Technologies, Business Process Interoperation.

\section{INTRODUCTION}

To react dynamically to changes is today's most desired property for production enterprises. There are several aspects to enterprise dynamic reconfiguration. In this paper we focus on reengineering production facilities in case of product redesign and in case of changing demand, and on optimizing the production process or removing errors that might have emerged.

The VIDOP project aim is to develop methods to combine distributed models of the components (sub-models) to build a complete model (integrated model) of the whole production facility. The final result is an infrastructure for vendor integrated decentralised modelling (IVM) which includes a communication platform enabling cooperative work with clearly defined views between the manufacturer and the suppliers to transmit securely and quickly models (geometric models, process 
models, workflow models), machine programs, documentation, notifications etc. The Internet allows the use of the provided models at distributed locations (manufacturer, supplier) leading to a decentralized and parallel optimization process with faster reaction to changes in product design or system errors at the manufacturer site.

This project is funded by the European Commission's "Competitive and Sustainable Growth" and is being developed by a trans-European consortium, whose coordinator is KUKA Schweissanlagen GmbH. Participants include: DaimlerChrysler AG and University of Karlsruhe, Germany, INGEMAT, S.A. and ROBOTIKER, Spain, EFACEC and University of Porto, Portugal, Tecnomatix, Israel, Turnkiek and Eindhoven University of Technology, Netherlands, and Methodos S.p.A., Italy.

This paper is organized as follows. Following Section describes an illustrative example to provide the motivation for our developments and introduces a brief description of the current practice. Later Sections present a conceptual architecture for cooperative production planning, its prototype implementation and finally a brief guided tour to the developed prototype.

\section{ILLUSTRATIVE EXAMPLE AND MOTIVATION}

Consider the following example from the automotive industry. An existing DC body shop in Sindelfingen (DE) has to be modified in order to be capable of delivering a new product with slightly different characteristics from the existing ones (different sunroof). The production of this product requires the modification of the actual manufacturing line. These modifications are analyzed internally by the OEM (DC). The project at hand involves reengineering of an existing line in order to handle new products along with the "old" ones. This project can be split into several tasks, either according to the cells (components) that need to be modified, or to the technologies involved in the process.

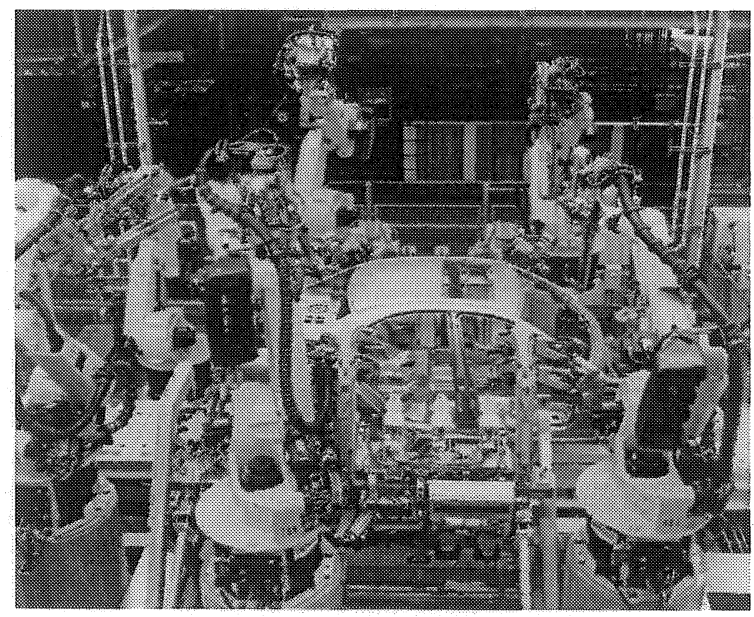

Figure 1 - Assembly cell from the body in Sindelfingen (DE) 
Usually, the OEM contacts the supplier of the existing line mostly because this supplier has all the required information (specification, models, etc.). This fact precludes the OEM from selecting a different vendor from its pool of usual "technology" suppliers (that best fit the tasks identified for this project).

In our example, the OEM selects the supplier of the original line (KUKA). KUKA, acting as a turnkey supplier, then selects sub suppliers to take care of the redesign of cells 1 to 3 and of the handling system. In turn, suppliers 1 and 3 feel the need to split their task (this is their project) into subtasks. Each of the suppliers then selects from their own pool of "technology" providers the ones that best fit the tasks at hand.

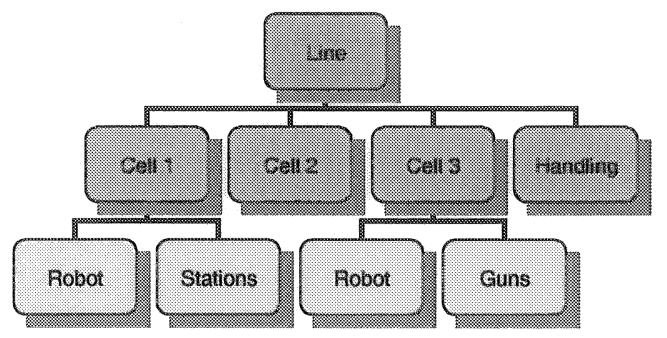

Figure 2 - Overall project structure (component view)

These temporary organizations - partnerships - only last for as long as the associated project and must be setup rather quickly. After the partnership is defined and tasks are allocated among the involved partners, the necessary information has to be exchanged (and information flows must be defined).

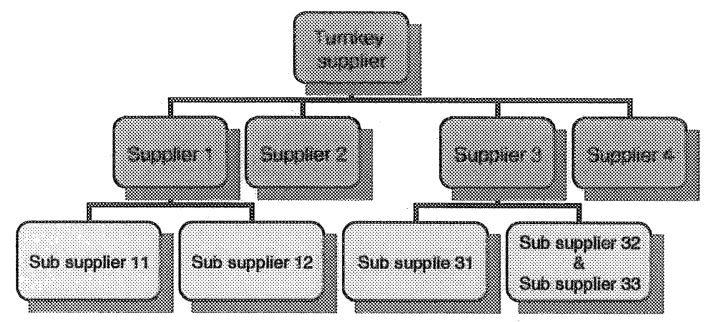

Figure 3 - Overall project structure (company view)

Information, drawings and models are exchanged on a need-to-know basis only. In this case, the OEM sends the necessary information (cell drawings, part drawings and new part parameters) to the turnkey supplier who, in turn, sends the information about cells 1 and 2 to suppliers 1 and 2, etc. Supplier 1 filters the information he has and only sends partial models and drawings to its sub suppliers (11 and 12). In the case of sub suppliers 32 and 33 they both get the same information but maybe with different privileges. 
The case of supplier 4 is special. In order to redesign the handling system for the overall line, supplier 4 needs information about the overall system (e.g. to perform material flow simulations). Supplier 4 needs information about cells 1 to 3 but not in the level of detail needed to redesign the cells. Although the "as is" information is necessary to start this task, supplier 4 also needs updated information about the changes planned to cells 1,2 , and 3 .

\section{Generalization}

Rooted on the particularities of the example presented and on the "Phase and Role Model" [1] a generalization of the "Cooperative Plant Production" (CPP) scenario can be developed. In this more general scenario we will have several OEMs and several technology suppliers (which can also work as sub suppliers) divided into Line Builders (suppliers of a complete line or cell, but also can work as turn key suppliers) and Engineering Houses (suppliers of partial solutions).

In a general case the OEM can subcontract a project (new line or line change) to a turnkey supplier (line builder), or to different line builders for different lines/cells (the OEM is responsible for project management), or to different line builders for different lines and different engineering houses for lines designed by OEM (the OEM is responsible for project management).

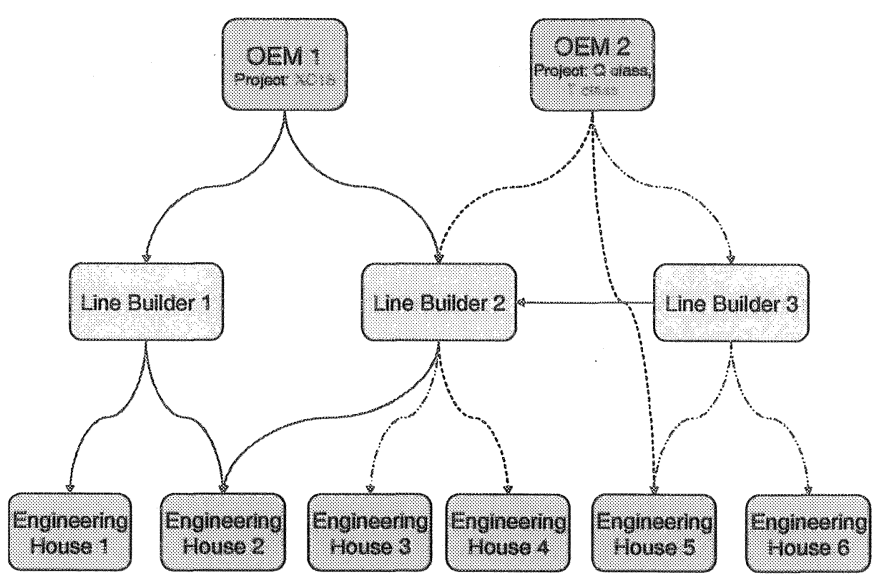

Figure 4 - Generalized Cooperative Plant Production

A Line builder can work as supplier for different OEMs and, at the same time, as turnkey supplier. A line builder can also work as sub supplier of other line builders. In turn, the line builder subcontracts work to other line builders and to engineering houses. An engineering house can work as subcontractor of different line builders and different OEMs. It can also be the case of working as a sub supplier of the other engineering houses. The roles and responsibilities of each of the partners in a generic project are presented in Table 1.

Currently, information potentially releasable to partners is often combined with other, sensitive data. The unfortunate result is a denial of useful information to 
cooperating partners. Filtering information into small, coherent, discrete packages (views) makes it easier to control and thus distribute to other partnership members.

To support this operational concept a new paradigm [2], that manages information in terms of standardized, discrete objects, is required. Such an approach would enable to:

- Filter information objects from their sources.

- Enable publish, subscribe, query, and transform data objects

- Specify the policy governing how to disseminate and access data objects.

This would be a system of systems that manages, integrates, aggregates, filters and distributes information to cooperating partners.

Table 1 - Roles and responsibilities

\begin{tabular}{|c|c|}
\hline Role & Responsibilities \\
\hline $\begin{array}{l}\text { OEM / } \\
\text { Turnkey } \\
\text { supplier }\end{array}$ & $\begin{array}{l}\text { Manage and control planning process for OEM project scope } \\
\text { Integrate different sub models into complete model } \\
\text { Planning activities and optimization of complete model } \\
\text { Reuse of processes and resources } \\
\text { Keep core competency and know how for manufacturing and } \\
\text { planning } \\
\text { Keep flexibility in selection of line builder }\end{array}$ \\
\hline $\begin{array}{l}\text { Line } \\
\text { builder }\end{array}$ & $\begin{array}{l}\text { Manage and control planning process for line builder project } \\
\text { scope } \\
\text { Provide OEM with sub process models for integrated OEM } \\
\text { environment } \\
\text { Integrate different sub process models of engineering houses } \\
\text { Integrate different sub simulation models of engineering houses } \\
\text { Reuse own standards and best practices }\end{array}$ \\
\hline $\begin{array}{l}\text { Engineering } \\
\text { houses }\end{array}$ & $\begin{array}{l}\text { Manage and control planning process on engineering house } \\
\text { level } \\
\text { Deliver to line builder / OEM the sub process models or the sub } \\
\text { simulation models }\end{array}$ \\
\hline
\end{tabular}

\section{Current Approach}

Today there is no common infrastructure to support this kind of cooperative projects. Communication flows are very well defined but information management is done on a project basis, and communication is done through dedicated lines using proprietary platforms. This makes it very difficult for small sized enterprises to collaborate with different OEMs because of the burden associated with information management, and the cost associated with the required platforms.

As a consequence, reaction time is very slow, which means that ultimately there is a longer time to market, and there is a huge possibility of redundant information exchange and inconsistent information. At the same time, the security and access control methods used are strong cooperation hinders. 


\section{CONCEPTUAL ARCHITECTURE FOR CPP}

\section{Organizational Concept}

Based on the generalized "Cooperative Plant Production" scenario a supporting conceptual organization can be derived. The proposed organization is based on a hierarchical recursive structure.

The OEM sets up a project, identifying a task and a partnership, (one in the case of a turnkey supplier) to several suppliers, to work on the project. In turn, each partner creates a new project based on his task (or tasks). These new tasks are either done in house or (sub) suppliers are contacted to deliver the product.

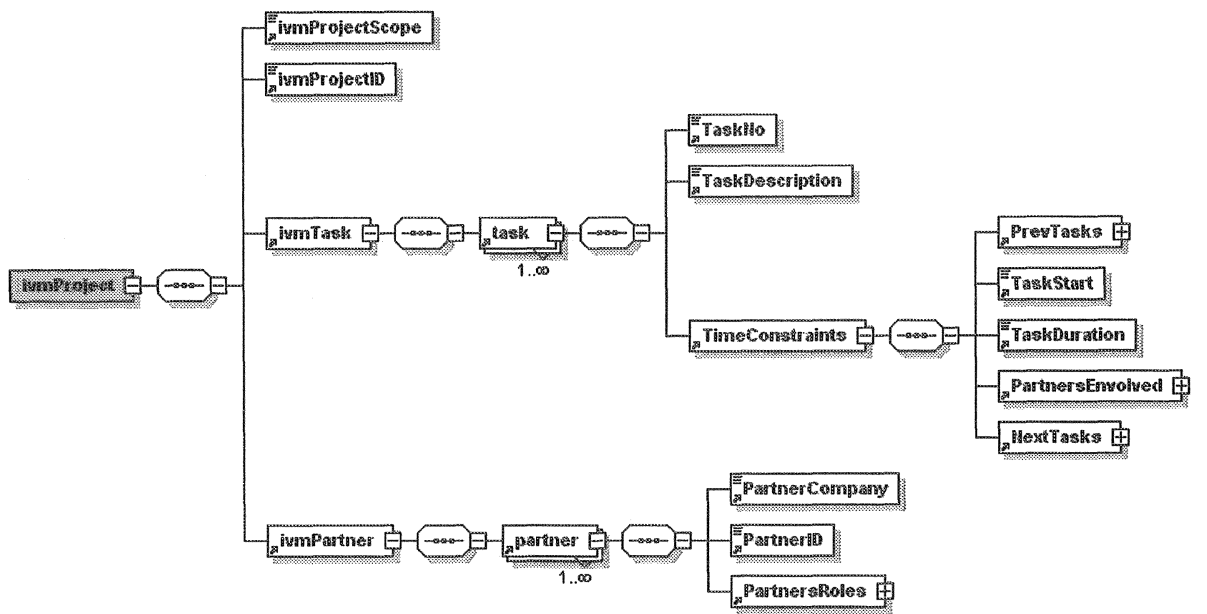

Figure 5 - CPP Schema

This organization structure can be defined using an object oriented approach (Figure 6) or using XML. Figure 5 represents the XML schema for the conceptual organization presented. Besides partners and tasks, the sequence of tasks, their duration and precedence relations, the companies and their role, are also defined.

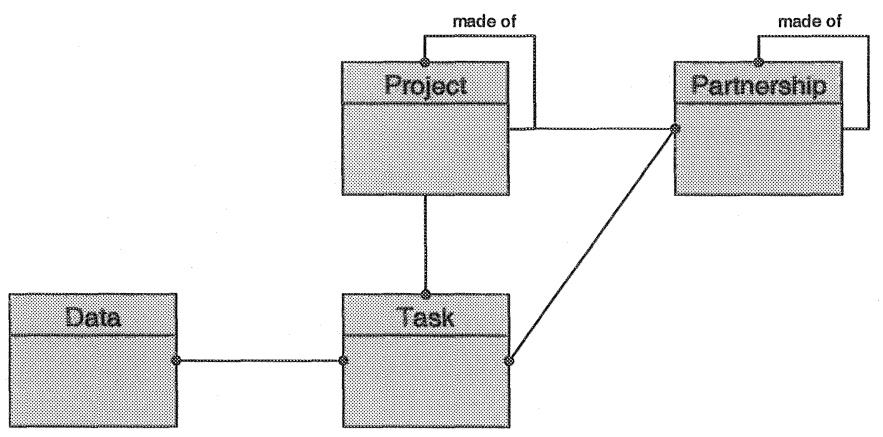

Figure 6 - Model for co - operative project 


\section{Methods and Rules}

In order to enhance existing cooperation in plant production, methods for the exchange of information (models or just data, etc.) and events have to be established. Some are merely administrative functions to establish and to manage cooperative work projects (defining tasks and work items), but others include user functionality for managing work and information.

Besides specific cooperative work functionality, cooperation cannot exist without trust and security. The building blocks of trustworthy cooperations are methods for secure communication and for information control.

Cooperation enabling methods and rules can be divided in three groups:

ఐ Cooperative work support methods.

- Workflow, work management and work sharing methods.

wnowledge protection and security methods.

Cooperative work support methods include such functionalities as:

- File sharing: exchange of initial data; download; upload; update.

- Collaborative work \& discussion: chat room; instant messaging; collaborative review of documents, images or models; video conferences.

* Outliner: collaborative environment for sharing ideas or organizing projects.

- Workflow definition and synchronization: task list definition; schedule definition: appointments; deliverables.

- Project management: (re)define user roles or access rights; status check.

For workflow management: work management and sharing rules can be classified as workflow dependent rules. These rules define special rules dependent on the process that is running, the company organization and on the methods that are used. For a cooperation project dependant cooperation workflow controls the intercompany flow.

The knowledge protection and security methods include functionalities to:

- Control access to shared data, with role based access control and customizable views.

- Authenticate and validate users, using two factor authentication.

- Secure data transmission over insecure lines, ensuring the privacy of the data.

- Ensure messages are not altered during transmission.

\section{Inter Company Workfllow}

According to the ISO 9000 [3], every organization exists to accomplish valueadding work. Work is accomplished through a network of processes. Business processes define the ways organizations add value.

The Workflow Management Coalition (WfMC) defines workflow as the complete or partial automation of business processes. A Workflow Management System is a system that is capable of defining, managing, and executing workflows.

In "Co-operative Plant Production" the network of processes spans across inter departmental and inter company boundaries. To enhance this feature, inter departmental and inter company workflows must be supported. Business processes 
must be combined in order to create process chains that transfer (or create) value, and since the integrated approach is not possible most of the times - process chains that span multiple organizations and workflows supported by different WMS - it is necessary to ensure workflow interoperability. Workflow interoperability is achieved when two or more workflow engines coordinate their activities in such a way that it looks like, for an external observer, a single service.

In 1994 the WfMC defined a reference model [4] that proposes an architecture for WMS. In this model five interfaces are defined, in order to insure that interoperability among workflow engines and other applications is possible.

For workflow engine interoperability, and hence for business processes interoperability, the used interface is number four. Through this interface heterogeneous workflow engines (from different vendors) can exchange, transparently, work items between themselves. This way it is possible to have several workflow engines cooperating in order to deliver a single workflow service.

This interoperability is achieved through the use of standards. Currently WfXML [5] is in use, and it is the result of a process that started with the "Workflow Interoperability Standard" [6], jointFlow, and SWAP (Simple Workflow Access Protocol). Wf-XML is a structured, well-formed XML based messaging protocol, independent of the transport mechanism (HTTP by default, but a SOAP binding is under development). Context data can be included through XML extensions.

\section{ARCHITECTURE}

The architecture for inter-company workflow management and for business processes coordination can follow a federated approach. In this approach, a central service is responsible for registering and locating processes and workflow engines. This central service can also be used to ensure workflow engine interoperability when direct interoperability is not possible.

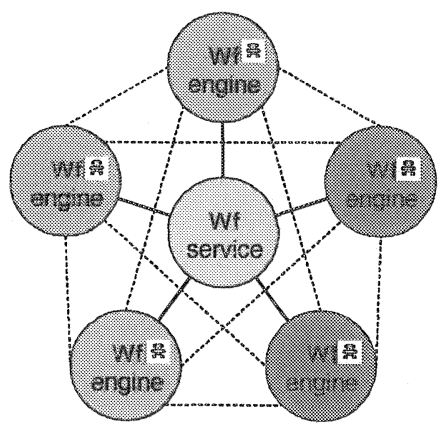

Figure 7 - Federated Workflow Architecture

The implementation of this architecture should use standards like $\mathrm{XML}^{10}$, SOAP ${ }^{11}$ and Wf-XML. The communication between the central service, which also

${ }^{10}$ eXtensible Markup Language 
works as lookup and registry, and the workflow engines can be achieved through webservices [7]. When supported by both ends, Wf-XML is the default communication protocol. However, Wf-XML is not yet widely supported over the major Workflow Management Systems (WfMS), so direct interoperability is now on project's background.

Since most of cooperative work lies on a worklist basis, the central service runs a WfMS which is responsible for assigning tasks to users in different companies. These users may manage their tasks (get the worklist, acquire and finish activities) locally by using a client application which sends predefined requests to the central service.

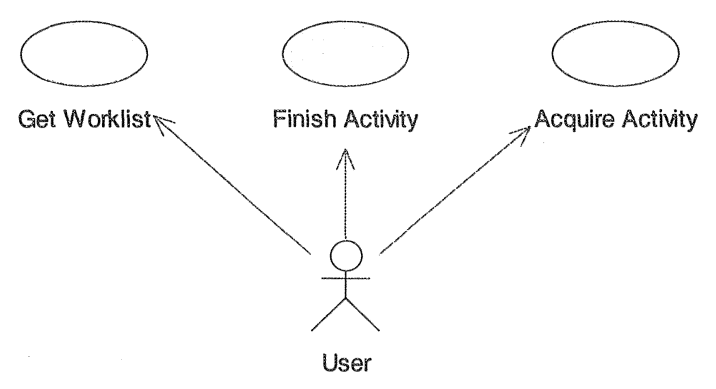

Figure 8 - Workflow Use-cases

As stated above, these requests use SOAP as the main encoding protocol for the Remote Procedure Calls. The WebService on the central service uses the API provided by the WfMS to communicate with it. This means that the WebService and the WfMS may be away from each other (using HTTP as the main transport mechanism). All the responses sent back to the user lay on a predefined XML format (and not just sending back the response supplied by the WfMS).

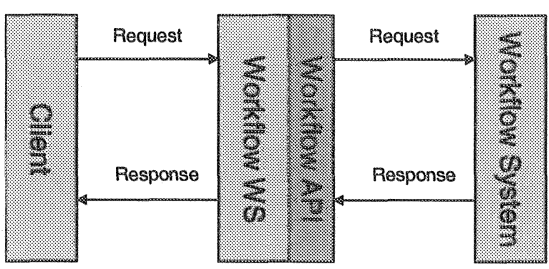

Figure 9-IVM Workflow top-view

The main idea here is that the WebService may painlessly switch the WfMS, as long as there is a communicating class for it, which implements the interface that defines the main operations. For now, these operations are just the ones declared above (get worklist, acquire activity and finish activity). The finish activity operation usually sets a final status to an activity, although there can be several final statuses available. The process instance will continue and the next activity depends on the status applied to the previous one.

\footnotetext{
${ }^{11}$ Simple Object Access Protocol
} 


\section{Prototype implementation}

The existing prototype already includes most of the security functionalities. Some basic data views and workflow functionalities are already implemented but further enhancements are on the way.

This prototype is based on a distributed architecture, with a local implementation for each participating partner and with a federated server, where the workflow service and some basic model management service are located.

The Wf Service, located at a federated server, is a workflow engine with a webservice on top of it. This workflow engine enacts processes associated with CPP. Rules of cooperation and interaction are stored on this engine along with process templates.

When a new project is created, these templates can be used as a basis to define the project structure, communication and information flows. The ways these templates are designed and stored are of WfMS' exclusive responsibility. The task management on the local client is basically implemented.

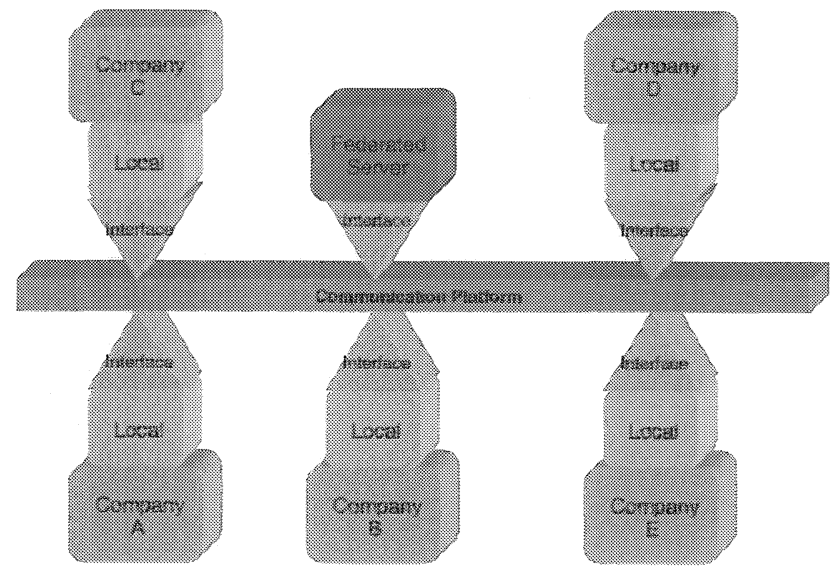

Figure 10 - Prototype Architecture

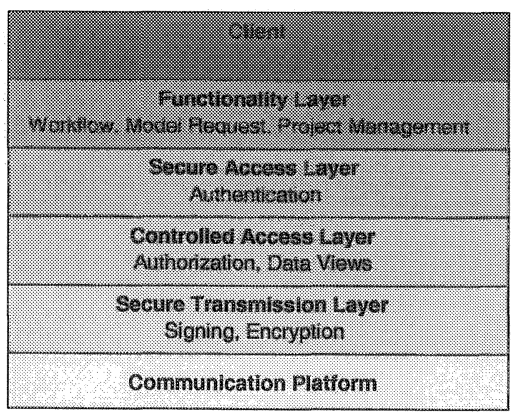

Figure 11 - Prototype Architecture (layers)

The client can send a request to get the user's worklist and display all the info related to each task, and then waiting for some user interaction. All the requests are 
Remote Procedure Calls which use several parameters, needed both by the WfMS and by the security layer. The WebService then translates the request to WfMS' API and waits for the response. It then parses that response and creates a new one based on a pre-defined client readable format and sends it back to the client.

Each local client uses a stack of functionalities that ensure the authentication, authorization and security. The prototype was implemented in JAVA using jdk1.4 and the webservices were implemented using Axis $^{12}$ (beta2) running on top of Tomcat ${ }^{13}(4.0 .3)$ servers.

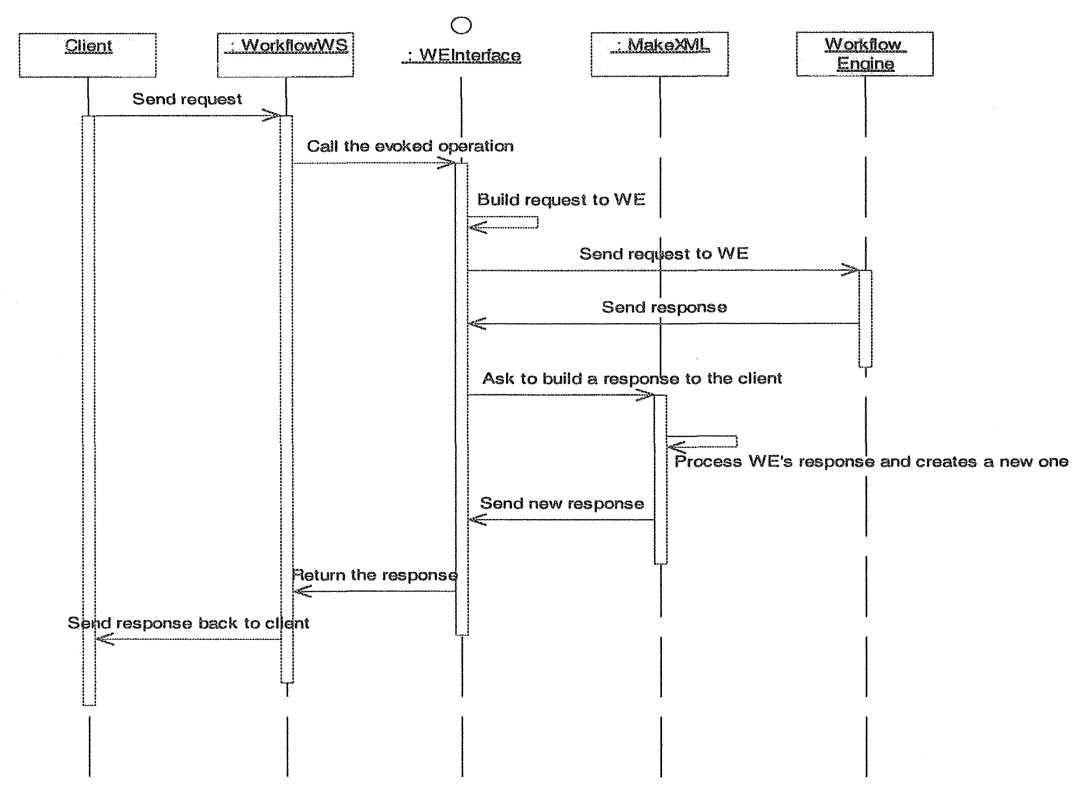

Figure 12 - IVM Workflow sequence diagram

\section{Security Layer}

The security layer it's responsible for ensuring several security aspects: authentication, authorization, data integrity and privacy, and non-repudiation. This layer it's divided in two main applications. One is composed by several webservices that provide authentication, authorization; and a second one that provides data integrity and privacy and non-repudiation [8].

In the implementation of the security layer, where possible, was implemented with security XML standards [9]. This choice enables a greatest portability of this layer.

\footnotetext{
${ }^{12}$ http: //xml.apache.org/axis/

${ }^{13} \mathrm{http}$ : //jakarta.apache.org/
} 


\section{Security Webservices}

The first one it's composed of several webservices that in conjunction with UI interfaces provides authentication, authorization, data integrity and privacy, and non-repudiation.

Regarding authentication, it's based on a two-factor. This system has to be flexible in order to accommodate the legacy two-factor authentication systems that are already install. Authentication is a very important aspect because it's how someone or some system identifies himself. Because of this, the system used has to be known to the partners that let you connect to their IVM. They can set a minimal requirement in terms of the authentication system used in order to let one IVM connect to theirs.

Authorization plays an important part in this kind of systems because it controls who can access to each company data. This data it's filtered accordantly to the views that the data owner gives to each of its partners. To take full advantage of this feature data has to be represented as XML so filters defined as XSLT ${ }^{14}$ can be applied. This way the data actually sent to the requester partner it's filtered respecting the rules defined by the data owner. The rules apply not to actual users but two pairs company/role. This pairs can be seen in the CPP schema (Figure 5).

Regarding the other aspects of the security layer, they are only implemented in the interface of the company's IVM to the public network. For now the company's IVM it's considered as a secure space, so no encryption on the communication is used. There will be an option to enable signing or encryption by the users.

\section{Security Gateway}

The Security Gateway it's positioned at the interface of the company's IVM to the public network. It works as a gateway where all IVM data will pass before entering or leaving the company's IVM. This gateway works at the SOAP level. That is, it processes SOAP messages. To ensure authenticity, integrity, privacy and non-repudiation every SOAP message that arrives from the company IVM it's sign and encrypted and every message that arrives from the outside has to have the same characteristics. For the gate to do these operations it has to have three main parameters:

- The company's key-pair and certificate;

- The destination company public-key certificate;

" The destination company Security Gateway.

The first it's used for signing of the SOAP message giving it authenticity, integrity and non-repudiation. The second is used to encrypt the SOAP message providing privacy to it. The third gives the physical destination of the partner Gateway.

When a Gateway receives a message tries to use it's private-key to decrypt it. If this operation it's not successful the message it's rejected. If not, it verifies the signature and validates the authenticity of the certificate used against the sender. If

\footnotetext{
${ }^{14}$ XML Stylesheet Language Transformations
} 
this step is ok, the Gateway tries to find the nature of the request and then forwards it to the proper service in its IVM.

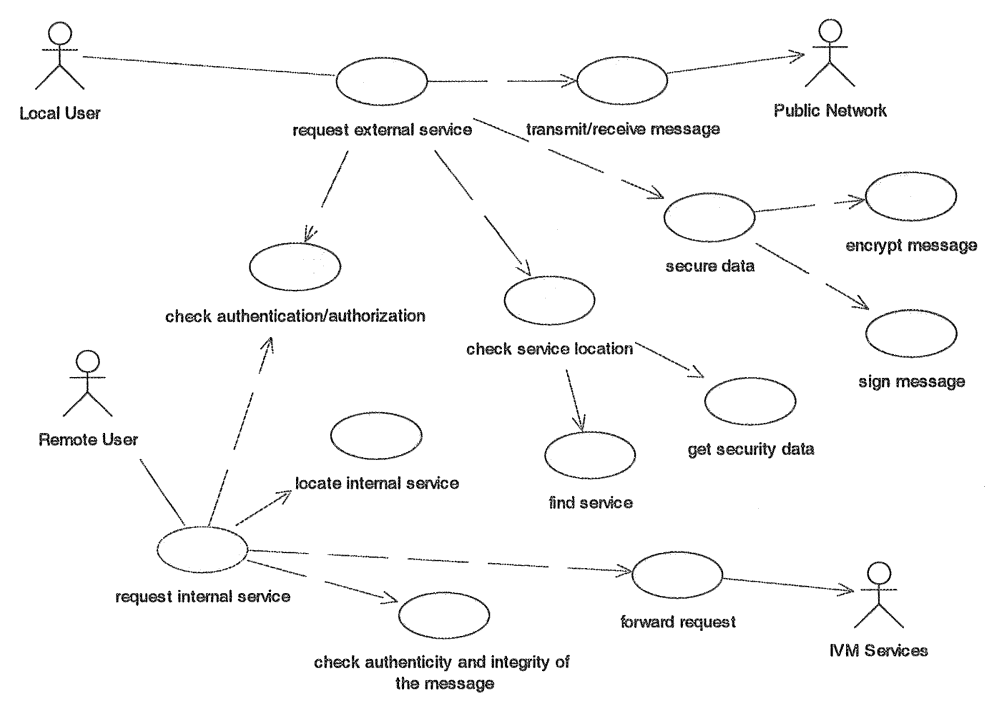

Figure 13 - Security Gateway use case diagram

\section{GUIDED TOUR}

The IVM workflow client offers some basic features to manage tasks. Looking at the front panel (Figure 14), one can see that the Workflow WS endpoint can be pointed out, although it is stored in a properties file.

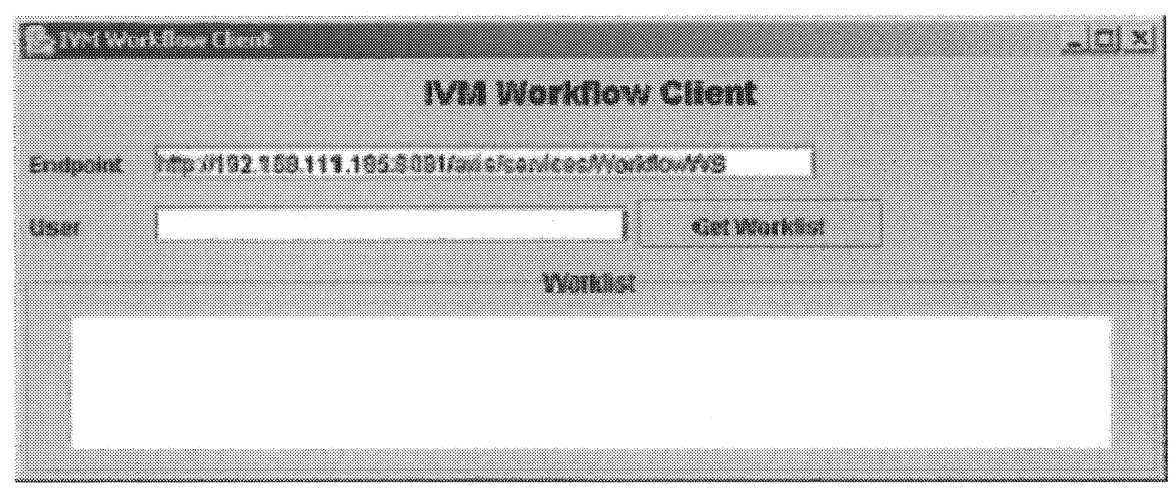

Figure 14 - IVM Workflow Client

There's no login sequence since this client will be fitted in the final IVM Client which will, of course, have an authorization/authentication system. So, one can just insert a user name and that's all. This user name must be registered on the WfMS 
and there's no need for password administration by now. The user can use the "Get Worklist" button to retrieve his worklist which is refreshed automatically every minute. On the next screenshot (Figure 15) there's an example of a worklist.

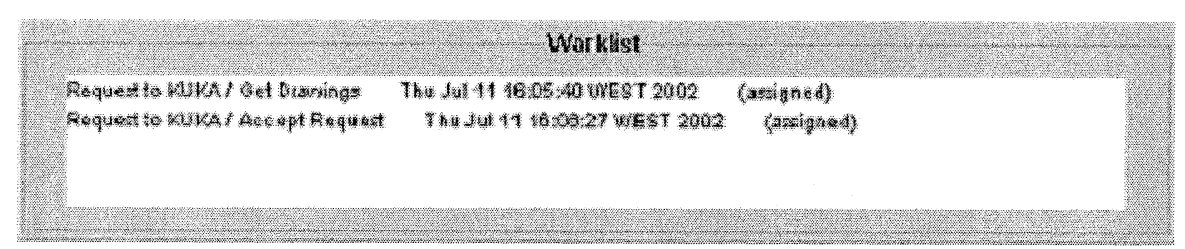

Figure 15 - Worklist example

After the user select an activity, a panel with all the activity related info shows out (Figure 16).

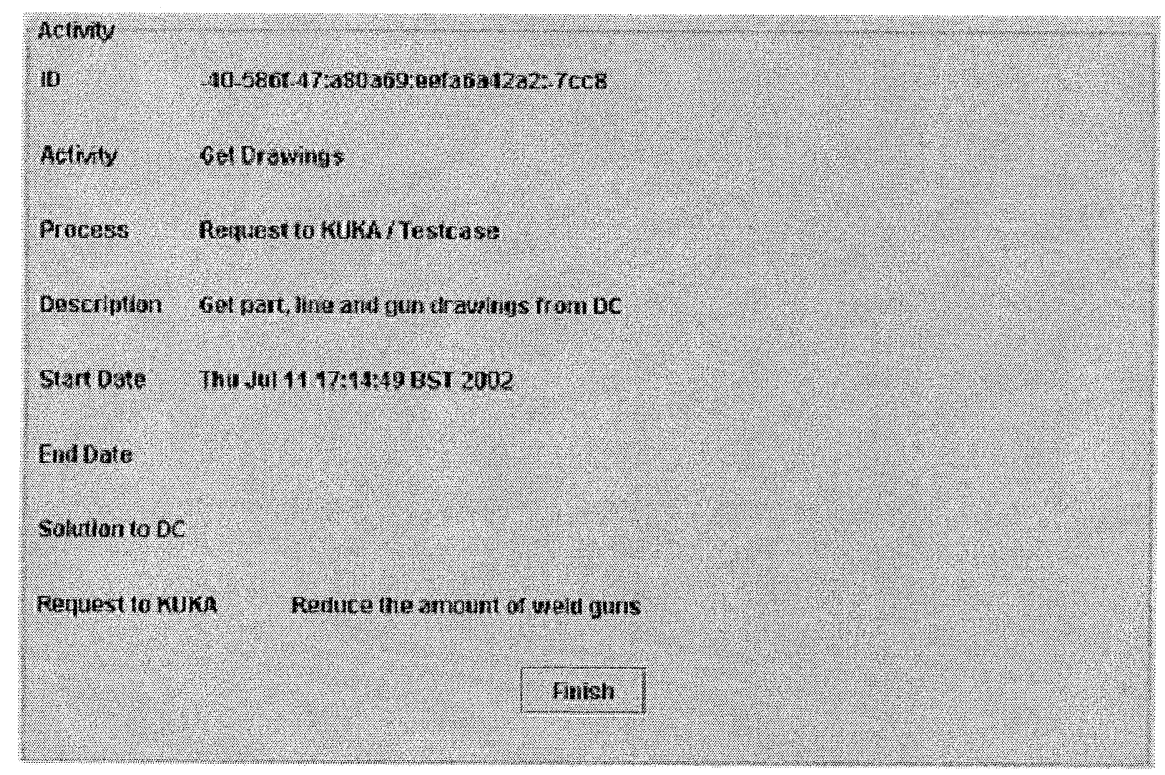

Figure 16 - Activity Panel

At the bottom of the activity panel, the user may use one of the buttons to set a status to the activity and finish it. If there's no activity specific statuses defined, there's always a default status available: "Finish". After finishing the activity, the worklist is refreshed. If there's not an "assigned" flag on some activity entry in the worklist, it means that that activity is assigned to several users and one must acquire it first. If so, there will be an "acquire" button at the activity panel when that activity is selected. 


\section{CONCLUSIONS AND FUTURE WORK}

This paper presents a general architecture for e-cooperation to address problems of enterprise collaboration and coordination. The proposed architecture defines the interactions and responsibilities among the parties involved in collaborative projects. Part of this work was developed in light of the VIDOP project, a trans-European project supported by the European Commission's GROTH Programme.

This general architecture is applied to case study drawn from the automotive industry. In this case study the architecture is mapped onto an infrastructure that is used to support the cooperative design and construction of production facilities. In this Cooperative Production Planning (CPP) project, several suppliers are involved in a project to reengineer a body shop. This application is very helpful in showing the main advantages of the architecture:

- Intra enterprise coordination and collaboration functionalities.

- Inter enterprise workflow interoperability functionalities.

- Cooperative work support methods.

- Security and knowledge protection methods.

A small description of a prototype implementation is also discussed. This prototype was built using the web technologies, like JAVA, XML, SOAP and WfXML.

Currently, the main focus of the work is moving onto the automatic selection (or evaluation) of potential partners and on the automatic definition of rules for cooperation (including operational rules - language and semantics - and business rules).

\section{ACKNOWLEDGMENTS}

This work was partially supported by the European Commission's "Competitive and Sustainable Growth Programme" (GROWTH) under the project VIDOP Vendor Integrated Decentralised Optimisation of Production Facilities (G1RDCT2000-00301).

\section{REFERENCES}

[1] Woerner, Jan and Laengle, Thomas and Woern, Heinz, Corporate Planning and Simulation of Plant Production Facilities in the Virtual World, Proceedings of the 18th International Conference on CAD/CAM, Robotics and Factories of the Future CARs\&FOF'02, Porto, Portugal, 2002, pp. 109 - 116.

[2] Marmelstein, Robert E., Force Templates: A Blueprint for Coalition Interaction within an Infosphere, IEEE Intelligent Systems, vol. 17, n. 3, 2002, pp. 36- 41 .

[3] International Standards Organization, Quality Management and Quality Assurance Standards, ISO, 1994.

[4] Workflow Management Coalition, The Workflow Reference Model (TC-1003), WfMC, 1995. 
[5] Workflow Management Coalition, Interoperability Wf-XML Binding (TC-1023), WfMC, 2000.

[6] Workflow Management Coalition, Interoperability Abstract Specification (TC-1012), WfMC, 1999.

[7] Snell, J. and Tidwell, D., Programming Web Services with SOAP, O'Rilley, 2002.

[8] Schneier, Bruce, Secrets and Lies: Digital Security in a Networked World, John Wiley \& Sons, 2000.

[9] XML Security Page <http://www.nue.et-inf.uni-siegen.de/ geuerpollmann/xml_security.html>, July 2002. 